\title{
École et société : pour une éthique de la liberté
}

Henri Peña Ruiz

\section{OpenEdition}

Journals

Édition électronique

URL : http://journals.openedition.org/ries/4167

DOI : 10.4000/ries.4167

ISSN : 2261-4265

\section{Éditeur}

Centre international d'études pédagogiques

\section{Édition imprimée}

Date de publication : 5 mars 1995

Pagination : 95-109

ISSN : 1254-4590

\section{Référence électronique}

Henri Peña Ruiz, «École et société : pour une éthique de la liberté », Revue internationale d'éducation de Sèvres [En ligne], 05 | 1995, mis en ligne le 16 mars 2015, consulté le 01 mai 2019. URL : http:// journals.openedition.org/ries/4167 ; DOI : 10.4000/ries.4167

Ce document a été généré automatiquement le 1 mai 2019.

(c) Tous droits réservés 


\title{
École et société : pour une éthique de la liberté
}

\author{
Henri Peña Ruiz
}

\section{Le monde comme il va}

1 Station de métro parisienne. Un homme jeune, "sans domicile fixe ", tente de s'endormir sur la banquette. Au-dessus de lui, l'immense publicité d'un magasin : « On aurait tort de s'en priver ».

Voici un principe de l'art de l'éducation que particulièrement les hommes qui font des plans d'éducation devraient avoir sous les yeux: on ne doit pas seulement éduquer des enfants d'après l'état présent de l'espèce humaine, mais d'après son état futur possible et meilleur, c'est-à-dire conformément à l'Idée de l'humanité et à sa destination totale. Ce principe est de grande importance. Ordinairement les parents élèvent leurs enfants seulement en vue de les adapter au monde actuel, si corrompu soit-il. Ils devraient bien plutôt leur donner une éducation meilleure, afin qu'un meilleur État pût en sortir dans l'avenir... (Kant).

2 À la conscience du malheur, le monde comme il va devient certainement très étrange. Sommes-nous à ce point habitués à l'inacceptable? Un instant, trop bref peut-être, la révolte du cœur voudrait détruire les décors où semblent passer, comme indifférents, les figurants éphémères de la multitude. Et crier, en plein jour, comme s'il s'agissait de mettre fin à ce sommeil éveillé où l'absurde passe inaperçu. L'imaginaire publicitaire étale sa débauche de richesses, de désirs rageurs et de violence jubilatoire. Par endroits, les yeux d'un enfant du Tiers-monde, comme un rappel dérisoire. « À votre bon cœur ».

On se souvient de la révolte de Rousseau. Comment les instruments de la puissance et de la richesse peuvent-ils côtoyer, dans le monde des hommes, le malheur et la souffrance? Paradoxe du " progrès » qui devrait conduire non à invalider le savoir et les techniques, mais à réfléchir sur le sens de leur utilisation sociale. Refus du pharisianisme, de la bonne conscience : l'exigence morale n'est pas pour l'auteur du Discours sur les sciences et les arts (1752) le supplément d'âme d'un monde sans âme, mais le retour à soi d'une humanité aliénée à ses propres dérives. Il y a « l'amour de soi » - bien différent de l'amour-propre - 
qui fait que chaque homme tend légitimement à faire ce qui lui permet de survivre. Recto, si l'on veut, de la force de vivre. Mais son « verso » n'est autre que la répugnance à voir souffrir autrui, « pitié » en un sens bien particulier, qui met en jeu la présence à soi d'une humanité dont chacun se découvre porteur. Source indélébile du sentiment moral. Ce qui advient à autrui m'advient à moi-même : le regard qui se porte aux larmes lorsque la vive souffrance de l'autre déchire la vie machinale de l'habitude prend le sens d'un réveil. Porteuse d'universalité, la conscience morale advient en deçà des pensées captives où fausses raisons et demi-savoirs tendent à différer toujours le moment de la lucidité. Comme Rousseau, Kant pensait que l'action morale, en son principe, n'a besoin d'attendre ni le savoir ni la philosophie. Il y a eu des actions bonnes absolument, en leur visée constitutive, avant qu'on en élucide le fondement. L'entreprise philosophique d'intelligibilité rationnelle ne prétend pas inventer la morale, mais assurer l'identification critique de ce qu'elle est réellement - si les mots ont un sens -, afin que les sophismes et les subtilités de la mauvaise foi n'en brouillent pas indéfiniment le sens et la portée. Déceler dans la conscience commune un principe de l'action morale - la bonne volonté, par exemple -, ce n'est nullement faire fi des réalités qui semblent démentir une telle assurance. Ni souscrire à une sorte d'optimisme unilatéral où la foi en l'homme se préserverait des tragédies de son histoire. Refusant de séparer la politique et la morale (voir : Émile), Rousseau veut justement penser ensemble, dans l'union de leurs différences, les deux dimensions essentielles de l'humanité : bonté nue, immanente aux processus qui peuvent la recouvrir et l'oublier, mais disponible malgré tout à ses réactivations dans la lumière vive de la "conscience"; corruption et dégradation d'un devenir où l'homme s'échappe à lui-même au point de s'habituer aux paradoxes d'un progrès mortifère (guerres, injustices et hypocrisie généralisée ne semblent-elles pas aller de pair avec l'extension des richesses, de savoirs et du pouvoir ?). L'humanité, capable du meilleur et du pire ? On pourrait le croire, à observer les trésors d'ingéniosité déployés pour blesser et tuer (« la guerre, c'est faire l'impossible pour que des morceaux de fer entrent dans la chair vivante ») mais aussi pour soigner, sauver, réconforter (voir l'action humanitaire de multiples associations).

4 C'est à ce point sans doute que l'exigence d'intelligibilité prend tous ses droits. Le constat de Rousseau ne se bornait pas à dresser l'une en face de l'autre les deux dimensions de l'humanité. Le monde comme il va semble marquer le triomphe de la mauvaise part. Mais ce qui est n'épuise pas ce qui devrait être, ni ce qui pourrait être. Traduire le pessimisme historique en pessimisme anthropologique, c'est fataliser le malheur. Pas plus que le fait ne dit le droit, les mœurs ne disent la morale.

5 Rousseau croit pouvoir faire appel du malheur des hommes en assignant ses causes dans des dérives complexes, où le sens de la vie se perd dans cela même qui devait l'affirmer. Le souci moral, s'il est authentique, ne peut plus dès lors se satisfaire d'un moralisme de l'exhortation et de l'édification, où se donnerait à connaître l'impuissance affirmée devant l'injustice foncière d'un monde humain abandonné à une logique supposée irrépressible. La vogue éthique et la vague humanitaire, si elles devaient occuper tout le terrain, signeraient une sorte de renoncement à faire du monde des hommes un monde humain : panser des plaies sans remonter à la cause des blessures. Avec, à la longue, la lassitude dont s'assortit la banalisation du mal. Et peut-être l'indifférence devant la multiplicité des sollicitations qu'une société en déshérence suscite de façon réitérée.

6 Dans sa radicalité, l'inquiétude morale invite l'homme à se ressaisir de son histoire, c'està-dire à se savoir libre. Libre de faire ou de ne pas faire, de consentir ou de refuser, de 
traiter l'humanité comme une fin et non seulement comme un moyen. Libre, donc responsable, chaque fois que l'homme peut décider du sens qu'il donne à sa vie. La guerre, l'injustice, la tromperie, la solitude dans la foule, la misère au sein des richesses, ne sont pas des maux extérieurs, qui adviendraient à la façon d'un cataclysme naturel. Il est étrange comme on peut se dire "dépassé par les machines"-retombant dans ce fétichisme sans âge qui prête aux choses un pouvoir dont les hommes seuls sont détenteurs. L'argent fait des petits, le marché fait des palais et des ruines, la machine fait des chômeurs. Dure loi d'un monde maléfique où se meuvent des hommes hébétés, scrutant les cours de la Bourse ou les offres d'emploi comme jadis on guettait dans le ciel les oiseaux de l'espoir. Désenchantement moral et nouvelles superstitions des fétiches économiques... Le monde comme il va vide les mots en les multipliant, comme il tue le sens dans le vertige d'une communication qui se prend pour fin. L'éthique, paraît-il, s'empare des entreprises, au moment même où des plans massifs de licenciement s'y annoncent. Jésus avait cru devoir chasser les marchands du Temple, et Marx confronter les proclamations morales avec la logique d'un système qui les noie dans «les eaux glacées du calcul égoïste » (Manifeste de 1848). Difficile liberté, à déchiffrer en sa possibilité toujours vive malgré ce que Sartre appelait le «coefficient d'adversité des choses». L'exigence éthique s'est-elle perdue - et disqualifiée à tout jamais - dans le moralisme dérisoire dont s'assortit une société civile rivée à ses calculs et aux puissances qui la rançonnent ? La question doit être posée, comme une sorte de préalable où le travail de la pensée s'interdit tout angélisme: les bons sentiments vont trop souvent de pair avec l'affairisme pour que le premier souci de la conscience critique ne soit pas de refuser ce type de conformisme.

7 Rousseau se tourne vers la politique, et l'éducation. Par la première, il croit devoir envisager une « refondation » de la société. « Principes du droit politique » : le sous-titre du Contrat Social dit suffisamment ce qu'il en est de sa démarche. Dire le droit, c'est aussi ne pas consentir à ce qui est. La lucidité requiert que l'on explique comment ce qui est a pu advenir comme il est. Mais le rappel à soi de la liberté humaine indique l'irréductibilité de la volonté politique si prompte aujourd'hui à s'effacer devant l'invocation des contraintes du réel. On sait que Rousseau, penseur de la République, solidarise égalité et liberté : «c'est justement parce que la force des choses tend toujours à détruire l'égalité que la force de la législation doit toujours tendre à la maintenir ${ }^{2}$ ». Par l'éducation, Rousseau entend faire appel de cette corruption qui ne semble devenir la loi des hommes qu'en raison de la soumission servile à l'air du temps. Éduquer à la liberté, ce n'est pas préparer des petits conformistes, bien « adaptés » au monde comme il va, mais édifier une humanité libre qui, en chaque homme, puisse faire que le donné corrupteur soit transcendé. Un homme libre est celui qui est l'auteur de ses pensées et de ses actes : la faculté de se donner à soi-même sa propre loi signe, dans l'ordre éthique comme l'ordre politique, la véritable autonomie. La pesanteur du donné - préjugés, mœurs, traditions, de tous ordres - ne peut dès lors être invoquée contre ce qui fait qu'un homme est un homme, à savoir sa liberté même. Kant développera la force d'une telle idée en rappelant qu'il n'est de moralité que l'homme libre, et de l'homme raisonnable. Agir moralement, ce n'est pas imiter un modèle - car il reste à comprendre pourquoi l'action exemplaire est exemplaire - ce qui requiert la raison comme saisie du principe de l'action. Aucun monde réel - fût-il de mensonge et de mal généralisés - ne peut rien prouver contre un tel idéal, en lequel l'humanité remonte à sa source, à l'idée de son accomplissement possible. En refusant de faire du monde comme il va la norme de l'éducation, Kant et Rousseau esquissent le pouvoir propre de tout ce qui permet de s'affranchir du donné. Une société 
peut-elle, spontanément, se mettre à distance d'elle-même? Ceux qui soulignent l'emprise des idéologies, des visions du monde, et des préjugés de l'heure, ne font qu'indiquer le statut de la liberté : conquête difficile, relevant, certes de sa propre possibilité inscrite en tout homme, mais aussi de ce travail sur soi qu'on appellera culture, si l'on veut bien concevoir sous ce dernier mot l'effort indéfini par lequel chacun s'élève à la liberté effective du jugement et de l'action, au-delà de toute soumission et de toute appartenance. Ici prend sens la question de l'École.

\section{Éthique et liberté : le pari de l'école}

8 Fonder l'école publique et laïque, c'est s'ordonner au vœu de Condorcet: «rendre la raison populaire ${ }^{3}$ ». Les principes mêmes de la République, dans une telle instauration, conduisent à reconnaître à l'école une autonomie relative par rapport à la société civile. D'abord, au niveau des finalités, si le but d'une éducation républicaine est de faire de chacun un sujet libre, dans ses pensées comme dans ses actes, aucune inculcation de valeurs ou de modèles n'est admissible. De ce point de vue, l'adaptation à la société du moment - mot d'ordre ambigu - ne peut signifier l'intériorisation de ses contraintes particulières. Les mimétismes serviles par lesquels l'école serait sommée de se plier au dernier cri de la modernité hypothèquent sa distance nécessaire - et partante, sa puissance libératrice. L'école publique, en ce sens, n'est pas un simple «service» qui devrait se borner à répondre à une " commande sociale ", mais une institution organique de la République. Celle-ci, comme le rappelle Montesquieu, a besoin de la puissance de l'instruction, car seuls des citoyens libres et éclairés peuvent assurer sa survie (le despotisme, lui, gouverne à la peur, et n'a cure de cette liberté éclairée). Cet « intérêt » de la République pour l'instruction et la liberté de tous ses citoyens lui font un devoir de ne pas laisser l'accès au savoir dépendre des disparités de fortune propres à la société civile. L'instruction sera donc publique, gratuite et obligatoire, afin que le droit qui lui correspond ne soit pas hypothéqué par d'éventuelles résistances familiales ou locales. Dire qu'une école ainsi définie n'est pas un simple service, c'est aussi rappeler son sens éthique et politique. Former le citoyen à la liberté, c'est lui donner pour assise une humanité aussi accomplie que possible. Si l'éducation vise le tout de l'homme, il est clair que l'instruction, finalisée par la liberté du jugement qui rend possible la lucidité de l'action, possède d'emblée une portée éducative. Mais ceci en un sens très précis, excluant tout endoctrinement moral : l'autonomie finale est toujours en jeu dans ce long processus où l'élève apprend à se passer de maître. La joie de comprendre, couronnant l'effort vers le vrai, donne à la personne une solidité morale autrement plus forte que les simulations incertaines de la spontanéité sans normes ou à l'inverse la mémorisation passive de formules catéchistiques. Mais qu'est-ce véritablement que comprendre? Non pas disposer d'informations multiples, mais s'élever à la connaissance réfléchie. L'esprit ne peut véritablement connaître que ce qu'il fait sien, intimement, comme le rappelait saint Augustin dans le De magistro. L'instruction n'est pas simple «transmission de connaissances", opération de transvasement d'une tête pleine à une tête vide. Bien comprise, elle met en jeu l'activité libre de l'esprit s'élevant aux principes des choses à partir des signes dont use le maître. Enseigner, c'est montrer et, de ce fait, présupposer en l'élève le pouvoir de reconnaître, d'identifier le vrai. Comme le dit Platon, chacun dispose de l'organe de la connaissance, métaphoriquement la vue : il lui suffit d'en faire bon usage, de le tourner dans la bonne direction. 
« N'enseigner que des vérités ». Selon Condorcet, le pari de l'école publique est un double pari : sur la raison et sur la liberté. Sur la raison, car la puissance du rationnel est aussi celle du raisonnable: un optimisme nouveau, qui renoue en un sens avec la confiance socratique et platonicienne. Nul n'est méchant volontairement, sinon par ignorance. Le désir de savoir est aussi désir de sagesse, si la recherche qu'il inaugure ne s'arrête pas aux connaissances partielles isolées, mais entend les situer, les comprendre en leur sens dans une quête inlassable de l'intelligibilité. Le demi-savoir des demi-habiles n'est que lumière confuse, scientisme à courte vue. C'est bien pourquoi l'horizon encyclopédique doit permettre d'apprécier le sens de chaque connaissance dans la conduite de l'existence, en la référant aux fins de l'action. "Je vois le bien qu'il faut faire, et je fais le mal ». Cette maxime de l'Évangile semble invalider toute ambition de fonder rationnellement l'éthique. Mais Condorcet - en un sens, comme Rousseau dans la préface de Narcisse rappelle que le savoir n'est jamais corrupteur par excès : trop de savoir ne nuit point ; seuls peuvent nuire des savoirs mal compris, mobilisés dans des projets de puissance et des ambitions de domination qui attestent, par eux-mêmes, un défaut de véritable sagesse. Platon rappelait que le tyran qui persécute en croyant assurer ainsi son bonheur prépare en fait sa propre perte : aveuglement du demi-savoir qui donne à l'esprit resté en chemin sa présomption. La vraie raison n'est pas simple calcul - souvent à courte vue mais faculté qui requiert, sans transiger, une intelligibilité assurée de ses propres fondements. Tâche infinie peut-être, comme l'affirme Husserl, mais principe de liberté au regard des clôtures dogmatiques prenant appui sur des savoirs isolés, non questionnés quant à leur sens. C'est placer très haut les exigences du rationalisme des lumières - et interdire à tout jamais - les lectures réductrices qu'a pu en faire le ressentiment clérical. Le pari simultané sur la raison et sur la liberté conduit Condorcet à exclure toute idée de catéchisme moral. Sur ce point, il s'opposera à ceux des Révolutionnaires qui croient utile une promotion explicite, par un enseignement adéquat, des nouvelles valeurs. Une sorte de catéchisme républicain, en somme, exaltant l'égalité, la liberté, et la fraternité, alors que Condorcet fait confiance aux seules lumières de la Raison, librement cultivées, pour « retrouver » les mêmes principes. Débat exemplaire, dont les échos se retrouveront dans l'entreprise de Jules Ferry et ses ambiguïtés mêmes.

À ce point de la réflexion, la difficulté propre à un enseignement de la morale dans l'école de la République prend toute sa dimension. Se faire un devoir de n'enseigner que des vérités, c'est fixer un idéal régulateur dont on comprend qu'il s'accorde parfaitement avec le respect de la liberté de conscience, c'est-à-dire avec la laïcité. La chose publique res publica - est commune à tous : elle ne peut faire partager que ce qui unit les hommes, ce à quoi nul ne peut opposer la singularité de ses croyances. Dans un espace plan, la somme des angles d'un triangle est égale à $180^{\circ}$. Une telle vérité, enseignée, ne peut choquer personne, du moins de droit. On sait que l'héliocentrisme copernicien choqua d'abord beaucoup d'hommes, mais la résistance de fait, offerte à l'astronomie scientifique, relevait de préjugés géo-centristes et finalistes. Des vérités peuvent déranger, provisoirement, mais à la façon dont l'exigence rationnelle peut déranger des croyances spontanées. L'école se doit de les enseigner dans la mesure où elle est le lieu où la raison apprend à s'affranchir, par un travail méthodique, de ce qui tout d'abord la détourne d'elle-même. Cette rupture-là est saine. Autre chose la sphère des convictions morales particulières, et des croyances religieuses: le souci de l'universel, du bien commun à tous, ne peut s'assortir d'aucune préférence accordée par exemple à une religion particulière, ou à une idéologie politique déterminée. Laos: le mot désigne le 
peuple en son entier, dont aucune partie n'est opposable aux autres. La "laïcité » maintient la référence à la totalité du peuple comme horizon obligé de toute parole enseignante. Lieu de l'universel en somme, l'école publique ne peut-être que laïque, sauf à faire violence à tel ou tel des élèves qu'elle réunit en son sein. L'éthique de la laïcité - car il y en a une - ressortit en fin de compte à la liberté de conscience, forme essentielle de la liberté, et à l'égalité la plus stricte des élèves - puisqu'aucune des croyances particulières qui sont par ailleurs libres de se déployer dans la société civile ne doit être promue par l'école publique, en tant qu'elle est l'école de tous. Dans les écoles privées au sens strict, celles qui sont nées du désir de tel groupe humain de faire cultiver ses valeurs particulières dans des lieux à cet effet, la question ne se pose évidemment pas: les croyances particulières promues par l'enseignement n'y peuvent choquer en principe aucun élève, sauf si celui-ci s'y trouve par violence. Reste à savoir si un monde commun de sens peut advenir entre des êtres qui seraient rivés à leurs appartenances, et seraient portés à s'enfermer dans les ghettos de leurs particularismes respectifs. On peut mesurer les risques d'une démocratie se bornant à juxtaposer des particularismes communautaires. Et les enjeux d'une démarche républicaine se proposant d'unir des individus sur la base de l'égalité des droits et des devoirs comme sur celle de la pleine liberté de conscience. Mais l'on mesure aussi, du même coup, les difficultés d'un tel principe d'unification, qui place toutes les institutions républicaines - et en premier l'École - dans une situation de tension vive par rapport aux multiples clivages qui déchirent la société civile, et aux puissances dominantes qui tendent à s'en dégager.

\section{Les tensions de l'histoire : le problème de la morale sans épithète}

11 Le généreux pari de Condorcet assumait l'exigence éthique sans lui reconnaître une spécificité différentielle par rapport à l'exigence rationnelle bien comprise. Il s'accordait d'emblée avec le principe de laïcité, sans avoir à s'embarrasser des questions touchant à la pluralité des visions du monde, que celles-ci soient religieuses ou non. Il présupposait une reconnaissance, par la société, du rôle propre de l'école, et de son incontestable vocation à faire advenir l'union des hommes par le partage des valeurs républicaines, elles-mêmes réinventées par chacun dans le libre exercice de sa raison. Un siècle plus tard, avec l'œuvre de Jules Ferry, les principes de Condorcet allaient rencontrer les tourments idéologiques du cléricalisme. Le vœu d'une école suffisamment affranchie des pressions sociales pour produire des effets propres de libération - et de promotion des principes républicains -, allait se heurter à l'hostilité du clergé, furieux de se voir dépossédé de son emprise sur l'institution scolaire. D'où les conflits, parfois très vifs et peut-être encore mal apaisés aujourd'hui, liés à l'avènement historique des principes fondateurs de l'école républicaine. Mais on conviendra en l'occurrence qu'il importe de distinguer nettement deux choses : d'une part le sens intrinsèque de ces principes - dont il s'agit de savoir s'ils sont fondés et justes ; d'autre part les souvenirs historiques associés à la reconnaissance mouvementée de ces principes -souvenirs qui peuvent sembler connoter les principes eux-mêmes de déterminations qui pourtant n'y sont pas contenues.

12 Le principe de laïcité, par exemple, ne stipule que la stricte séparation de la puissance publique et des croyances religieuses particulières, afin que celles-ci, libres de s'exercer dans la sphère privée, se retrouvent sur un plan d'égalité, comme il sied dans une 
véritable république, et puissent relever de la seule liberté de conscience, reconnue en sa plénitude. Une telle séparation, dès lors, n'a d'autre fondement que les droits de l'homme : égalité et liberté. Sa justification rationnelle ne fait intervenir à aucun moment l'hostilité à la religion; seul le cléricalisme, comme investissement de la sphère publique par une croyance particulière (qu'elle soit religieuse ou propre à une idéologie politique) se trouve en cause. Historiquement, entre 1880 et 1905, la crispation de certains dignitaires catholiques (Monseigneur Freppel par exemple) contre l'œuvre laïque a provoqué de vifs conflits: les Républicains se heurtèrent alors aux autorités ecclésiastiques, mais il serait faux d'attribuer de ce fait à la laïcité une essence antireligieuse. On peut rappeler à ce sujet le discours de Ferdinand Buisson (chrétien luimême) du 26 janvier 1903: "Nous n'entendons nullement faire la guerre à l'idée religieuse, encore moins supprimer l'idée religieuse. Ce que nous voulons combattre - et c'est le sens de notre vote - ce n'est pas l'idée religieuse, c'est l'idée ecclésiastique, l'organisation cléricale, c'est la tyrannie cléricale ». Bien avant Ferdinand Buisson, Victor Hugo lui-même avait développé cette distinction dans un discours célèbre du 15 janvier 1850 :

J'entends maintenir, quant à moi, cette antique et salutaire séparation de l'Église et de l'État, qui était l'utopie de nos pères, et cela dans l'intérêt de l'Église comme dans l'intérêt de l'État.

13 Cette mise au point devrait permettre de saisir dans toute leur actualité le sens et les ambiguïtés de l'articulation proposée par Jules Ferry entre instruction publique et éducation morale. La lecture de la Lettre de Jules Ferry aux instituteurs (17 novembre 1883), référée au contexte, permet de poser un problème dont les termes sont à bien des égards exemplaires, même s'ils requièrent aujourd'hui une transposition réfléchie à la lumière des évolutions les plus récentes. En toile de fond, tout d'abord, la polémique déclenchée par Monseigneur Freppel contre "l'école sans Dieu». Jules Ferry entendait fonder la gratuité et la laïcité de l'école sur les principes de 1789. Monseigneur Freppel considère que ceux-ci constituent « la négociation du péché originel ». Une campagne tenace contre l'école publique s'efforce d'accréditer l'idée qu'une école sans référence religieuse ne peut être qu'immorale. Se trouve donc mise en cause, très directement, la possibilité de fonder la morale sur la raison propre à chaque homme: l'instruction publique, solidaire du rationalisme des lumières, amalgamé abusivement au scientisme, ne pourrait avoir aucune véritable portée morale, et consacrerait même la propension d'une humanité orgueilleusement affranchie de la tutelle divine à se vautrer dans le péché. En face d'un tel discours, que peuvent faire et dire Ferry et les promoteurs de l'idée laïque? Deux lignes sont possibles. La première consiste à tenir bon sur l'idée que le rationalisme des lumières ne se réduit en aucun cas à l'apologie d'une raison scientiste, simplement capable de calcul scientifique ou d'ingéniosité technique. Cette ligne rappelle que la raison est aussi puissance éthique, faculté des fins et non simplement des moyens. Et ce, dans le droit fil de Rousseau et de Kant, mais aussi d'Auguste Comte, dont le positivisme est tout le contraire d'un scientisme. Nul besoin, dans ce rationalisme généreux, de souligner la faiblesse et la finitude de l'homme pour ensuite en appeler à une nécessaire transcendance religieuse comme unique fondement possible de la morale.

Si l'on entend par « capacité de transcendance » la faculté qu'a un homme de s'arracher à l'immédiateté de ses pulsions et de ses représentations, la raison immanente à l'homme enveloppe une telle capacité. Quel est le bien qui mérite d'être poursuivi pour lui-même? Question originelle de L'Éthique à Nicomaque d'Aristote. Question décisive à l'évidence pour toute fondation rationnelle de la morale. «Agir de telle façon que tu considères toujours 
l'humanité dans la personne d'autrui comme une fin et jamais simplement comme un moyen ». Maxime kantienne, qui signale le pouvoir qu'a un être de sentiment, de désir et de passion, de s'affranchir du calcul intéressé et des mobiles subjectifs pour respecter l'humanité comme telle. Être de raison et non seulement de désir l'homme manifeste alors la rectitude possible de son vouloir, rectitude qui n'attend pas que le cours du monde lui soit favorable pour se manifester absolument. Non par l'héroïsme de la raison, mais simple affirmation du pur devoir, en amont de toute argutie ou de toute supputation d'efficacité. Reconnaître à l'homme la puissance du raisonnable (et non seulement du rationnel), ce n'est pas affirmer, contre l'évidence, qu'il en use toujours: la moralité, selon la raison, est elle aussi conquête sans cesse réitérée - comme peut-être, si l'on peut oser le parallélisme - l'affirmation religieuse de l'esprit contre la captation des désirs de la chair. De fait, pour Rousseau, la raison éthique a aussi pour nom conscience, en laquelle spontanément se manifestent les bonnes dispositions de l'humanité. On le voit, une telle « ligne" philosophique ne manque pas d'arguments. Mais la question se complique dès lors que l'effectivité des inégalités sociales, des tutelles idéologiques et religieuses, des crispations politiques, induit des postures passionnelles qui font obstacle, de fait, à l'appréhension de ce rationalisme universaliste comme légitime fondement possible de la moralité. D'où une deuxième ligne, plus ambiguë, mais peut-être plus «facile» dans le contexte de l'époque, et qui prône un enseignement moral explicite, apparenté par sa visée universaliste à celui des connaissances, et distingué de toute promotion de croyances particulières. C'est cette deuxième ligne que choisit Jules Ferry, au risque peutêtre de brouiller l'idée même d'instruction publique, en la mêlant à celle d'éducation nationale (le terme est employé) et morale. Revenons au texte de la Lettre aux Instituteurs, pour en pointer les tensions internes. D'abord, l'affirmation dépourvue de toute ambiguïté du partage requis par l'école républicaine :

...distinguer deux domaines trop longtemps confondus: celui des croyances, qui sont personnelles, libres et variables, et celui des connaissances, qui sont communes et indispensables à tous, de l'aveu de tous.

D'autre part, la revendication, pour l'école laïque, d'un rôle moral :

L'instruction religieuse appartient aux familles et à l'Église, l'instruction morale à l'école.

On remarquera ici l'introduction d'un concept qui peut paraître problématique : celui d'« instruction morale», sans doute forgé par analogie avec l'instruction civique. Mais quel peut bien être l'objet de cette instruction morale ? Enseigner des règles, ce n'est pas encore s'assurer de leur mise en œuvre effective. Surtout si une telle mise en œuvre requiert une disposition durable à agir de telle façon plutôt que de telle autre (vertu comme habitus, au sens aristotélicien du terme). Un enseignement proprement théorique, exposant par exemple les différentes doctrines morales et faisant réfléchir sur leurs présupposés respectifs ne s'écarterait pas des exigences de l'instruction publique : assurer la connaissance des doctrines morales (ou religieuses), ce n'est pas les promouvoir ou les invalider. De ce point de vue, que l'on peut élargir en pensant aux débats actuels, la connaissance précise des mythologies, des religions, et des diverses représentations morales n'a rien à voir avec une "sensibilisation religieuse" ou un endoctrinement moral, ou si l'on veut avec une éducation morale ou religieuse. Or sur ce point, le vocabulaire de Jules Ferry est significativement flottant, puisque les expressions éducation morale et instruction morale sont données pour équivalentes pour définir le contenu de «l'enseignement moral». On se souvient que pour Condorcet, si l'instruction peut avoir une portée éducative, c'est justement en s'assumant comme telle, c'est-à-dire en cultivant 
la faculté propre à la raison de s'élever, par la connaissance, aux principes du savoir et de l'action. Dans ce cas, la finalité éducative n'est atteinte que par la médiation d'une instruction, mais ne requiert pas un autre type de médiation qui, par exemple, jouerait sur les sentiments ou l'imitation de modèles.

Au moment de définir les modalités de l'instruction morale, Jules Ferry dissimule à peine son embarras, qui contraste avec le ton d'évidence adopté pour poser la nécessité d'une telle instruction. Qu'on en juge, à partir des deux extraits qui suivent :

... il a paru tout naturel que l'instituteur, en même temps qu'il apprend aux enfants à lire et à écrire, leur enseigne aussi les règles élémentaires de la vie morale qui ne sont pas moins universellement acceptées que celles du langage et du calcul

... j'ai dit que votre rôle, en matière d'éducation morale, est très limité. Vous n'avez à enseigner, à proprement parler, rien de nouveau, rien qui ne vous soit familier comme à tous les honnêtes gens.

18 Si l'on songe aux vives polémiques contre l'« école sans Dieu », taxée d'immoralisme par le ressentiment clérical, on comprend la prudence de Jules Ferry («rôle très limité »), inspiré par le principe de liberté de conscience, mais aussi la revendication d'universalisme, qui présuppose une sorte d'accord général sur les principes élémentaires de la conduite morale. Conjuguée à la volonté d'affirmer que la morale ne relève pas exclusivement d'un fondement religieux, la présupposition d'un tel accord va au-devant des objections déjà fréquentes selon lesquelles l'école laïque, comme telle, serait partisane. Jules Ferry esquisse ici cette fameuse « morale sans épithète », c'est-à-dire ce qui serait à ses yeux la morale tout court, sans qualificatif d'appartenance. L'école publique n'enseignerait ni une morale "catholique » ni une morale "athée », mais la morale, celle qui réside dans la rectitude commune des actions bonnes et honnêtes. Dans des accents qui peuvent rappeler la Profession de foi du Vicaire Savoyard de Rousseau, Jules Ferry évoque « la sagesse du genre humain » et le "patrimoine de l'humanité ». Les «bases philosophiques» d'une telle morale peuvent varier, mais il ne lui semble pas nécessaire d'en faire un objet de discussion :

... cette bonne et antique morale que nous avons reçue de nos pères et mères et que nous nous honorons tous de suivre dans les relations de la vie, sans nous mettre en peine d'en discuter des bases philosophiques.

19 Étonnante recommandation de la part d'un rationaliste soucieux de former l'esprit critique. Comment faire sienne une règle dont on ne discute pas le fondement ou dont, au moins, on n'élucide pas le caractère raisonnable? La vocation rationaliste de l'instruction publique, qui exclut tout principe d'autorité, peut-elle s'accorder avec l'idée d'une instruction morale s'abstenant d'aborder le fondement rationnel d'une norme ou d'un principe ? Jules Ferry résout, ou tente de résoudre la difficulté en alléguant un consensus possible sur les principes de la morale. La Lettre aux instituteurs poursuit en effet de la façon suivante :

Si parfois vous étiez embarrassé pour savoir jusqu'où il vous est permis d'aller dans votre enseignement moral, voici une règle pratique à laquelle vous pourrez vous tenir. Au moment de proposer aux élèves un précepte, une maxime quelconque, demandez-vous s'il se trouve à votre connaissance un seul honnête homme qui puisse être froissé de ce que vous allez dire (...). Si oui, abstenez-vous de le dire ; sinon, parlez hardiment, car ce que vous allez communiquer à l'enfant, ce n'est pas votre propre sagesse : c'est la sagesse du genre humain.

L'existence supposée d'un consensus semble ici dispenser de toute entreprise d'examen des «bases philosophiques ", entreprise périlleuse si, faisant apparaitre une pluralité de fondements possibles, elle ouvre la voie à une démarche relativiste et à la manifestation 
virtuelle de désaccords. On devine là encore les préoccupations conjoncturelles de Jules Ferry, mais peut-être convient-il de tenter une analyse des implications ambiguës d'un tel propos, tout en envisageant un cas de figure permettant de l'illustrer. Il a été noté un peu plus haut que la crainte d'une réflexion sur les fondements philosophiques d'un principe moral pouvait sembler paradoxale sous la plume d'un rationaliste convaincu - mais qu'elle ressortissait sans doute à un profil universaliste destiné à esquiver toute discussion sur des points controversés, donc à préserver la neutralité républicaine. Mais à quel prix se paye une telle esquive? Outre la tension ainsi introduite entre instruction et éducation dévolues à des modalités pédagogiques différentes, la présupposition du consensus risque d'amarrer l'école aux représentations dominantes de la société civile, alors qu'en son essence l'école doit cultiver une capacité de distanciation par rapport aux visions du monde et aux idéologies ordinaires, sauf à abdiquer sa fonction libératrice. S'il s'agit de ne choquer personne, doit-on renoncer à défendre un principe considéré comme juste dès lors qu'on peut lui opposer l'objection virtuelle d'un « bon père de famille »?

21 Un exemple particulièrement sensible, hier comme aujourd'hui, permet de mettre en évidence la difficulté. La morale en jeu dans les rapports entre les sexes ne peut, en droit, s'ordonner qu'au principe d'égalité de l'homme et de la femme, et appeler le respect et les normes de conduite traduisant dans les faits la reconnaissance de cette égalité. Faut-il renoncer à enseigner un tel principe sous prétexte que l'on peut pressentir l'objection que ne manquerait pas de susciter le machisme diffus d'une société civile encore mal acquise à l'égalité des sexes? Plus grave encore, l'enseignement des vertus de la «femme au foyer » ou des responsabilités de l'homme "chef de famille», peut-il être validé du seul fait que lui correspond, à un moment donné, un « consensus » relatif dans la société ? Et faut-il s'interdire de soumettre au principe de raison l'éventuelle justification de l'inégalité des sexes? Jules Ferry, grand admirateur de la Révolution française, n'ignorait sans doute pas la Déclaration des droits de la femme et de la citoyenne d'Olympe de Gouges, qui avait le mérite d'élargir encore la portée universelle des principes d'égalité et de liberté (la moitié du genre humain, tout de même...). À vouloir cultiver le consensus, trop de manuels d'instruction civique et d'éducation morale des "petites écolières » comme des petits écoliers, ont relayé au sein de l'école les représentations idéologiques dominantes de la société civile de l'époque : la " femme au foyer », le " chef de famille ", « la mission civilisatrice» supposée de la France coloniale, ont marqué les mémoires. Certains théoriciens hâtifs ont cru devoir en tirer l'idée que l'École n'était qu'un «appareil idéologique d'État ", ou un lieu de "reproduction » pure et simple : thématisation bien peu dialectique, et très injuste, car ne rendant pas du tout compte de la tension, installée au sein de l'école républicaine, entre la pression idéologique de la société civile, hébergée ou relayée dans certains cas par une éducation morale trop portée au conformisme et la dimension authentiquement libératrice de l'instruction rationaliste promue par Jules Ferry. Aujourd'hui, de nouveaux conformismes peuvent s'introduire dans l'école à la faveur de la fameuse « ouverture de l'école sur la vie » ou du mot d'ordre combien ambigu d'adaptation au mode de l'économie.

\section{La raison des principes}

22 S'il est clair que l'école ne peut jamais s'abstraire totalement des influences du monde social et économique de l'heure, il est non moins clair que sa fonction émancipatrice requiert que ses finalités propres soient reconnues comme telles, et avec elles la 
légitimité d'une distance salutaire à l'égard des sollicitations toujours pressantes des puissances du moment. Sur le plan éthique, comme sur le plan des connaissances, une telle distanciation sauve en un sens la liberté de l'élève, en l'affranchissant virtuellement de la domination des normes familiales ou sociales, ou du moins en lui permettant d'en entrevoir d'autres. Mais en la matière, la démarche de l'école laïque ne va pas de soi et le caractère problématique des recommandations de Jules Ferry le montre bien : n'est-il pas paradoxal que l'exigence de distanciation rationnelle et critique y soit mise entre parenthèses dès qu'est évoquée la question morale? En témoigne alors la modalité pédagogique privilégiée : celle de l'exemplarité pratique, dont le maître lui-même doit être porteur, et non celle de l'élucidation rationnelle des fondements. Le texte de Jules Ferry est à cet égard tout à fait explicite : «Il ne s'agit plus là d'une série de vérités à démontrer mais, ce qui est tout autrement laborieux, d'une longue suite d'influences morales à exercer sur ces jeunes êtres »... «Peu de formules, peu d'abstractions, beaucoup d'exemples ». Cette morale de l'exemple et de l'influence, voire de l'édification, rompt sur un point essentiel avec l'inspiration kantienne : qu'on se souvienne de l'avertissement de Kant dans les Fondements de la métaphysique des mœurs :

Il n'y aurait rien de plus funeste à la moralité que de vouloir la tirer d'exemples. En effet, quelque exemple qu'on m'en propose, il faut d'abord que le juge d'après les principes de la moralité, pour savoir s'il est digne de servir d'exemple original, c'est-à-dire de modèle (...). L'initiation est exclue de la morale, et les exemples ne peuvent servir qu'à encourager, en mettant hors de doute la possibilité de faire ce que la loi ordonne....

L'« exception pédagogique » envisagée pour l'éducation morale, par élision de la critique rationnelle qui remonte aux fondements, relève peut-être d'un souci stratégique lié à la conjoncture: Jules Ferry n'était peut-être pas encore assuré du rapport de forces pour oser soumettre à la raison la question éthique. Mais elle est lourde de conséquences, car à son insu peut-être l'auteur de la Lettre aux instituteurs prend le risque de légitimer, sous couvert d'universalisme mou - ou plutôt de consensus imaginaire - une tutelle idéologique imposée par la société civile du moment. Les implications dernières du principe de laïcité invalident une telle tutelle : c'est donc bien une véritable contradiction qui sous-tend la Lettre - et qui aujourd'hui encore - peut paraître exemplaire. Imaginons un instant la cohérence que réintroduirait la soumission de la question morale à la juridiction de la raison. La recherche raisonnée de l'Universel s'interdirait alors toute logique d'« influence », pour privilégier le libre examen des fondements. Il s'agirait alors d'un travail proprement philosophique, à entreprendre plus tard, avec des adolescents. Reprenons l'exemple kantien :

Agis de telle sorte que tu traites l'humanité, aussi bien dans ta personne que dans la personne d'autrui, toujours en même temps comme fin, jamais simplement comme moyen $^{5}$

Une telle maxime ne pourrait-elle recevoir, outre la justification que lui donne une fondation relevant d'un humanisme rationaliste (Kant lui-même), une légitimation mettant en jeu la règle d'or chrétienne ("Aime ton prochain ») ? Considérer l'humanité comme fin, ce peut être tout aussi bien voir en elle l'œuvre de Dieu, qu'il faut respecter, ou s'attacher à l'humanité comme telle, digne de respect. Deux actes de foi distincts peuvent être en jeu, mais la démarche rationnelle qui rend explicites les présuppositions auxquelles elle s'ordonne est cela même qui fonde la lucidité. Ce repérage critique de ce qui, en l'homme, est acte de foi, ne l'invalide pas, mais lui assure un statut conscient - donc susceptible d'être assumé ou rejeté comme tel. Deux validations distinctes d'un 
même principe moral sont ici concevables: la différence n'est pas en l'occurrence controverse inéluctable - et permet de dégager sans équivoque le niveau de réflexion auquel se situera le point d'accord. Un tel cas de figure est-il le plus fréquent? La question reste ouverte - mais il est permis d'entrevoir peut-être ici le statut d'une référence aux questions morales qui puisse s'accorder avec les exigences de l'instruction publique.

Il ne s'agit plus tant d'enseigner des règles morales que de mettre les élèves en mesure de réfléchir sur leurs fondements. Tâche philosophique par excellence. La raison ne s'interdit pas - ou plus - d'examiner les « bases philosophiques » de la morale - voire son statut problématique - dans une société dont la logique interne peut faire paraître dérisoires les proclamations éthiques. Mais selon l'exigence de vérité, il ne s'agit pas de le faire à la façon dont on débattrait d'opinions. Deux attitudes inverses et symétriques sont alors congédiées afin de dégager non pas des va leurs et des conduites uniformes mais une commune exigence de raison dans la capacité, dévolue à chacun, de fonder ses choix en connaissance de cause. La première attitude, présupposant un improbable consensus spontané, croyait pouvoir s'autoriser d'un universel déjà donné, et faire l'économie d'une mise à distance réflexive. Peut-être releva-t-elle chez Jules Ferry d'une conviction plus stratégique que réellement philosophique et pédagogique. La seconde attitude, procédant d'un relativisme radical, inviterait au seul débat d'opinions dans lequel on prendrait son parti des différences irréductibles de points de vue manifestés. La question est de savoir si sous prétexte d'invitation à la tolérance, un tel débat d'opinions n'a pas pour effet d'accréditer la dangereuse idée qu'en matière morale tout est affaire de simple opinion. Ce qui se donne comme relativisme libérateur peut souvent faire le lit de la tyrannie ou des pires violences de l'histoire. Car enfin le propos raciste n'est pas simple opinion, mais délit, et ce au regard de principes qui en quelque façon doivent bien transcender la relativité des dites opinions.

Congédier les deux attitudes, c'est esquisser le difficile chemin d'une morale de la liberté, c'est-à-dire tout à la fois d'une morale faisant de la liberté sa fin ultime et d'une morale misant sur la liberté de la pensée critique, cultivée en chacun comme seul «moyen » adéquat à la fin visée. C'est en liberté que l'élève vise la liberté comme accomplissement éthique de l'humanité. On voit toute l'exigence qui s'attache à une telle liberté de principe, qui est tout le contraire d'une spontanéité sans normes, bien vite asservie aux préjugés ordinaires. Dans une telle perspective, la liberté est travail, patience de la pensée rationnelle, conquête sans cesse réitérée d'un universel qui n'est pas donné, mais à construire. Cette conception critique et dynamique de l'universel fait apparaître le monde des opinions changeantes comme ce qu'il est: un kaléidoscope des représentations en lesquelles se figent des points de vue particuliers, des intérêts relatifs au moment, au lieu, à la position. Il ne s'agit pas alors d'en nier la consistance, mais d'en produire la conscience de soi, critique et distanciée, afin que s'éprouve en chacun tout à la fois la relativité de ce qui lui apparaîtrait, et la nécessité de s'en délivrer. Goûter, au moins une fois, cette sorte d'appel d'air d'une pensée affranchie - et comme délivrée des crispations quotidiennes - c'est peut-être s'élever à la responsabilité éthique la plus haute de l'humanité : le souvenir qui en résulte donne tous les courages au travail de la pensée. Le livre majeur de Spinoza intitulé Éthique déploie, sans concession, cette patience de la pensée qui sait jouir d'elle-même dans le moment précis où elle fonde aussi la difficile recherche d'une lucidité agissante. 

tintamarre et assoupie dans la banalisation de ses violences, peut encore reconnaître à l'École de la raison et de la liberté la possibilité de vivre sa propre distance. Et de réconcilier ainsi avec une certaine idée de l'humanité et de la morale ceux que le spectacle du monde pourrait bien plutôt faire basculer dans l'indifférence lasse ou la dérision sans principes.

\section{NOTES}

1. Kant, Réflexion sur l'Éducation, Vrin, 1993, p. 79-80.

2. Contrat Social, II, 11.

3. Premier mémoire sur l'Instruction publique, Garnier-Flammarion

4. Fondements de la métaphysique des mœurs, deuxième section, Bordas, p. 35.

5. Fondements de la métaphysique des mœurs, deuxième section, Bordas, p. 62.

\section{RÉSUMÉS}

La place éventuelle de l'exigence éthique à l'école n'est pas dissociable du rapport entre l'école et la société. Kant rêvait d'une éducation visant un monde meilleur, et l'humanisme rationaliste de Condorcet, penseur de l'instruction publique, se fiait au pouvoir de la raison humaine pour éclairer l'action. Jules Ferry, dans le contexte des vives oppositions que suscitèrent les réformes laïcisant l'école, a voulu assortir l'instruction intellectuelle d'une éducation morale. Le sens des difficultés qu'il a rencontrées est exemplaire, puisqu'il s'agit encore aujourd'hui de penser le rapport entre éthique et instruction rationaliste.

\section{INDEX}

Index géographique : France, Europe

Mots-clés : éducation morale, éthique, système éducatif, valeur, éducation

\section{AUTEUR}

\section{HENRI PEÑA RUIZ}

Professeur de philosophie, consultant au CIEP, France 